\title{
Atuação do enfermeiro na prevenção e tratamento do pé diabético em idosos: uma revisão integrativa de literatura
}

Nurse's performance in the prevention and treatment of diabetic foot in the elderly: an integrative

\section{literature review}

Desempeño de la enfermera en la prevención y tratamiento del pie diabético en los ancianos: una revisión de la literatura integrativa

Bárbara Leticia Corrêa Gomes

ORCID: https://orcid.org/0000-0002-9875-8981 Universidade Federal do Pará, Brasil

E-mail: barbaraltc4gomes@gmail.com

Paula Andreza Viana Lima

ORCID: https://orcid.org/0000-0002-8217-8288

Universidade Federal do Amazonas, Brasil

E-mail: paulaviana_lima@hotmail.com

Tainan Fabrício da Silva

ORCID: https://orcid.org/0000-0001-6761-1365

Universidade Federal do Amazonas, Brasil

E-mail: tainanfabricio@ hotmail.com

Natalie Kesle Costa Tavares

ORCID: https://orcid.org/0000-0002-0482-6999

Universidade Federal do Amazonas, Brasil

E-mail: natalietavares.nt@gmail.com

Thanaira Aicha Fernandes Maciel

ORCID: https://orcid.org/0000-0002-4119-6148 Universidade do Estado do Pará, Brasil

E-mail: aichafernandes1@gmail.com

Karina Raissa Rodrigues de Jesus ORCID: https://orcid.org/0000-0003-3221-7071 Faculdade Finama, Brasil

E-mail: karynarayssa@gmail.com Priscila Rodrigues Tavares

ORCID: https://orcid.org/0000-0002-0268-7200

Universidade do Estado do Pará, Brasil

E-mail: priscilatavares10@ hotmail.com

Jaqueline Ferreira Dias

ORCID: https://orcid.org/0000-0002-2224-3332

Faculdade Pan Amazônica, Brasil

E-mail: Jaquelinedias23@hotmail.com

Gabriela Nunes Pinheiro

ORCID: https://orcid.org/0000-0002-0126-3510

Universidade da Amazônia, Brasil

E-mail: james.silva008@gmail.com

Hellen Cristina de Oliveira Monteiro

ORCID: https://orcid.org/0000-0003-2345-1093

Universidade da Amazônia, Brasil

E-mail: hellenmonteiro24@hotmail.com 


\title{
Susi dos Santos Barreto de Souza \\ ORCID: https://orcid.org/0000-0002-4138-7147 Universidade Federal do Pará, Brasil E-mail: susis.barreto@yahoo.com.br Kátia Silene Oliveira e Silva \\ ORCID: https://orcid.org/0000-0001-6790-0394 Universidade Federal do Pará, Brasil E-mail: katiasilene@ufpa.br Felipe Costa Soares ORCID: https://orcid.org/0000-0003-4449-4324 Centro Universitário do Estado do Pará, Brasil E-mail: felipeesoaress@gmail.com \\ Djenanne Simonsen Augusto de Carvalho Caetano ORCID: https://orcid.org/0000-0001-7987-3178 Universidade do Estado do Pará, Brasil E-mail: djenanne.caetano@uepa.br \\ Francinéa de Nazaré Ferreira de Castilho \\ ORCID: https://orcid.org/0000-0002-9278-0010 Universidade do Estado do Pará, Brasil E-mail: francineacastilho@ hotmail.com Ivonete Vieira Pereira Peixoto ORCID: https://orcid.org/0000-0002-5463-9630 Universidade do Estado do Pará, Brasil E-mail: ivonete@hotmail.com
}

\begin{abstract}
Resumo
Objetivos: Identificar por meio de evidências cientificas a assistência de enfermagem na prevenção e tratamento do pé diabético em idosos no período 2008 a 2017. Metodologia: Este estudo consiste na pesquisa de revisão bibliográfica qualitativo desenvolvida por meio do método da Revisão Integrativa de Literatura, sendo realizada busca eletrônica em base de dados especializados em saúde e enfermagem. Utilizou-se as seguintes bases de dados: Banco de Dados em Enfermagem (BDENF), Scientific Electronic Library Online (SciELO), Literatura LatinoAmericana e do Caribe em Ciências da Saúde (LILACS). Resultados e discussão: A faixa etária evidencia que com o passar da idade esse público se torna mais suscetível a patologias crônicas com o aumento do processo de envelhecimento. O autocuidado é fundamental para a prevenção de lesões nos pés de portadores de DM, sendo difícil adesão por exigirem mudanças de hábitos e costumes, e pela negação que o indivíduo sente sobre a possibilidade de ser afetado pelas complicações dessa doença. Conclusão: As práticas educativas são a maior ferramenta para contribuir para a prevenção do pé diabético e consequentes amputações, possibilitando assim, sensibilizar os indivíduos para o desenvolvimento de habilidades para o autocuidado e mudança do estilo de vida.
\end{abstract}

Palavras-chave: Pé diabético; Idoso; Enfermeiro.

\begin{abstract}
Objectives: To identify through scientific evidence nursing care in the prevention and treatment of diabetic foot in the elderly from 2008 to 2017. Methodology: This study consists of a qualitative literature review research developed through the Integrative Literature Review method, and an electronic search was performed in a specialized database in health and nursing. The following databases were used: Nursing Database (BDENF), Scientific Electronic Library Online (SciELO), Latin American and Caribbean Literature on Health Sciences (LILACS). Results and Discussion: The age group shows that with age this public becomes more susceptible to chronic pathologies with the increase of the aging process. Self-care is fundamental for the prevention of foot injuries in patients with DM, being difficult to support because they require changes in habits and customs, and by the denial that the individual feels about the possibility of being affected by the complications of this disease. Conclusion: Educational practices are the greatest tool to contribute to the prevention of diabetic foot and consequent amputations, thus enabling individuals to sensitize individuals to the development of self-care skills and lifestyle change.
\end{abstract}

Keywords: Diabetic foot; Old man; Nurse.

\section{Resumen}

Objetivos: Identificar a través de evidencia científica la atención de enfermería en la prevención y tratamiento del pie diabético en las personas mayores de 2008 a 2017. Metodologia: Este estudio consiste en una investigación cualitativa de revisión de la literatura desarrollada a través del método de Revisión De la Literatura Integrativa, y se realizó una búsqueda electrónica en una base de datos especializada en salud y enfermería. Se utilizaron las siguientes bases de datos: Base de datos de enfermería (BDENF), Biblioteca Electrónica Científica en Línea (SciELO), Literatura Latinoamericana y del Caribe sobre Ciencias de la Salud (LILACS). Resultados y Discución: El grupo de edad muestra que con la edad este público se vuelve más susceptible a las patologías crónicas con el aumento del proceso de envejecimiento. El aut acariciamiento es fundamental para la prevención de lesiones en los pies en pacientes con DM, siendo difícil de apoyar porque requieren cambios en los hábitos y costumbres, y por la negación que el individuo siente sobre la posibilidad de ser afectado por las complicaciones de esta enfermedad. 
Conclusion: Las prácticas educativas son la mayor herramienta para contribuir a la prevención del pie diabético y las consiguientes amputaciones, permitiendo así a las personas sensibilizar a las personas sobre el desarrollo de las habilidades de cuidado personal y el cambio de estilo de vida.

Palabras clave: Pie diabético; Anciano; Enfermero.

\section{Introdução}

O Diabetes Mellitus (DM), é a patologia crônica que mais cresce, principalmente nos países em desenvolvimento. Destaca-se pela gravidade das suas complicações e também é considerado um problema de saúde pública em virtude do crescimento e envelhecimento populacional, da maior urbanização, da crescente prevalência de obesidade e sedentarismo, assim como da maior sobrevida dos pacientes diabéticos (Ferreira et al., 2020).

De acordo com Cubas et al. (2013) no Brasil, o controle do DM se dá por um conjunto de ações de saúde, individual e coletivo, que abrangem a promoção e a proteção da saúde; a prevenção de agravos; diagnóstico, tratamento, a reabilitação e a manutenção da saúde. Nesse domínio de atenção cabe à equipe multiprofissional o atendimento ao diabético, no entanto, o desenvolvimento de atividades educativas e o estabelecimento de estratégias para favorecer adesão ao tratamento são de competência do profissional enfermeiro.

A neuropatia sensitivo-motora acarreta perda gradual da sensibilidade tátil e dolorosa que torna os pés vulneráveis a traumas, denominada de "perda da sensação protetora". Um indivíduo diabético com perda da sensação protetora poderá não mais sentir o incômodo da pressão repetitiva de um sapato apertado, a dor de um objeto pontiagudo ou cortante no chão ou da ponta da tesoura durante o ato de cortar unhas. Acarreta, também, a atrofia da musculatura intrínseca do pé, causando desequilíbrio entre músculos flexores e extensores, desencadeando as deformidades osteoarticulares (Caiafa et.al., 2011).

Deste modo, o enfermeiro tem uma importante função na orientação dos cruciais, cuidados para os portadores de DM para impedir o surgimento de lesões ulcerativas, porém, por não ser o profissional que auxiliar orientações este grupo de pessoas e pela necessária importância de oferecer orientações educativas para a prevenção de ulceras surgiu o interesse em descobrir quais as orientações oferecidas por este profissional a portadores de DM, e se o portador está realmente informado do seu dever no próprio autocuidado (Marques et.al., 2019).

Barros afirma que (2012), a neuropatia é a complicação crônica mais comum e mais incapacitante do diabetes a mesma pode leva a perda da sensibilidade responsável por alerta o paciente danos que venham se agravar, essa redução da sensibilidade está diretamente ligada ao risco de amputação em consequência da falta de controle glicêmico, sobre peso, nível elevado de triglicérides e fatores biomecânicos que posam favorecer o aparecimento de lesões em membros inferiores de portadores de DM.

O pé diabético é caracterizado pela presença de pelo menos uma das seguintes alterações: neurológicas, ortopédicas, vasculares e infecciosas. Diante desse contexto, a participação do enfermeiro no atendimento ao paciente com DM, assim como da equipe multiprofissional, é vital para o restabelecimento e/ou manutenção da saúde do indivíduo portador de DM (Caiafa et.al., 2011).

Mediante ao exposto, a consulta de enfermagem é de fundamental importância não apenas no atendimento ao paciente com diagnóstico de diabetes, mas também nos casos nos quais há uma predisposição ao aparecimento dessa patologia. Portanto, através da consulta de enfermagem, o profissional passa a conhecer a história pregressa e socioeconômica do indivíduo, podendo assim montar um plano de cuidados específicos para cada situação (Ministério da Saúde, 2013).

Para evitar as complicações do Diabetes, é fundamental que haja a prevenção e que os profissionais de enfermagem devem acompanhar periodicamente o tratamento dos pacientes, orientando-os minuciosamente sobre os cuidados que deve ser tomado em relação ao controle glicêmico, à alimentação adequada, à prática de exercícios físicos e principalmente o autocuidado com os pés, procedimentos esses que serão capazes de permitir uma vida mais saudável (Carvalho, 2016). 
Portanto, delimitou-se como objeto de estudo a assistência de enfermagem no contexto da prevenção e tratamento do pé diabético em idosos, pois se trata de um processo, cuja importância e aplicabilidade são bastante discutidas na atualidade. Diante do exposto, o presente trabalho tem como objetivo identificar por meio da literatura científica nacional como se faz a assistência de enfermagem na prevenção e tratamento do pé diabético em idosos, bem como, caracterizar o idoso portador da doença, fatores de risco associados e as principais intervenções de enfermagem.

\section{Metodologia}

Este estudo consiste na pesquisa de revisão bibliográfica qualitativo desenvolvida por meio do método da Revisão Integrativa de Literatura (RIL). Inicialmente foi delimitado o tema da pesquisa sobre a Assistência de enfermagem na Prevenção e Tratamento do Pé Diabético em Idosos.

Se a evolução do Diabetes Mellitus pode gerar complicações como o Pé Diabético em razão da patologia instalada, a ausência das orientações prestadas pelo Enfermeiro na atenção básica é um dos maiores agravantes para o desenvolvimento de lesões de membros inferiores e agravos da doença. Desta forma, a carência de orientações para o autocuidado com os pés transmitida pelo enfermeiro para com o paciente, tende a levar o idoso a situações mais graves, podendo ocasionar a imobilidade, internação prolongada e até a perda parcial ou total do membro.

Portanto, diante desta perspectiva, este estudo traz como pergunta de pesquisa: $1^{\circ}$ Quais as intervenções de Enfermagem dispostas na literatura para prevenção e tratamento do pé diabético em idosos com DM, no período de 2008 à $2017 ?$

A pesquisa foi realizada por criteriosa busca eletrônica em base de dados especializados em saúde e enfermagem. Sendo assim, a pesquisa se utilizou das seguintes bases de dados: Banco de Dados em Enfermagem (BDENF), Scientific Electronic Library Online (SciELO), Literatura Latino-Americana e do Caribe em Ciências da Saúde (LILACS).

Para a realização desta pesquisa foram selecionados descritores não controlados (palavras-chave) e controlados. Os descritores controlados que foram utilizados neste estudo, obtidos no Decs, envolveram: "idoso"; "diabetes mellitus"; "educação em saúde"; "pé diabético", ‘cuidados de enfermagem" e "enfermagem", foram utilizados como descritores não controlados (palavras-chave): "Prevenção" "Tratamento", "Diagnóstico", "atuação do " enfermeiro", "intervenções de enfermagem", "hiperdia".

Para este estudo, utilizou-se como critérios de inclusão: artigos publicados em língua portuguesa; pesquisas disponíveis na íntegra e online; artigos escritos no período de 2008 a 2017, nas modalidades de pesquisa de campo, relato de experiência e dissertações e revisões integrativas. Como critério de exclusão utilizou-se: artigos que não estejam publicados na língua portuguesa; pesquisas que não foram disponíveis na íntegra e online; artigos escritos no período inferior a 2008.

A coleta de dados foi realizada por meio do Formulário de URSI, validado em 2005 (URSI, 2006). O instrumento apresenta cinco itens. Entretanto, para o estudo em questão o instrumento foi adaptado, o qual contemplou os seguintes tópicos: Identificação (título do artigo, autores, base de dados e ano de publicação); e Características metodológicas do estudo (tipo de publicação, objetivo e síntese dos resultados encontrados)

Para uma melhor compreensão deste trabalho, a análise preliminar do material bibliográfico foi realizada através de leitura exploratória que definiu quais textos se enquadraram à temática. Com a coleta realizada nas bases de dados, foram encontrados 3.220 artigos distribuídos entre três bibliotecas virtuais supracitadas e observa-se o número de artigos agrupados conforme os descritores e palavras-chaves. A leitura e análise integral dos artigos condicionou a seleção para 14 artigos que obedecem aos critérios de inclusão e exclusão pré-estabelecidos.

Para análise dos dados foi utilizado a análise de conteúdo de Bardin. Segundo Bardin (2009) este tipo de análise é um conjunto de técnicas que consistem em descobrir os núcleos do sentido que compõem a comunicação por meio de uma 
descrição objetiva e analítica. Para a autora, as fases de análise de conteúdo organizam-se em três pólos, os quais são: 1) Préanálise; 2) Exploração do material; e 3) Tratamento dos resultados: inferência e interpretação.

\section{Resultados e discussão}

\subsection{Identificação dos Artigos Analisados}

No Quadro 1, observa-se a identificação dos artigos em relação aos títulos, autores, base de dados e ano de publicação. Percebeu-se que 27\% (no total de 04) dos artigos foram retirados da base de dados LILACS, enquanto 36.5\% (no total de 5) artigos foram retirados da base de dados SCIELOe 36.5\% da base de dados BDENF (no total de 05).

Quadro 1: Identificação dos artigos selecionados para a Revisão Integrativa.

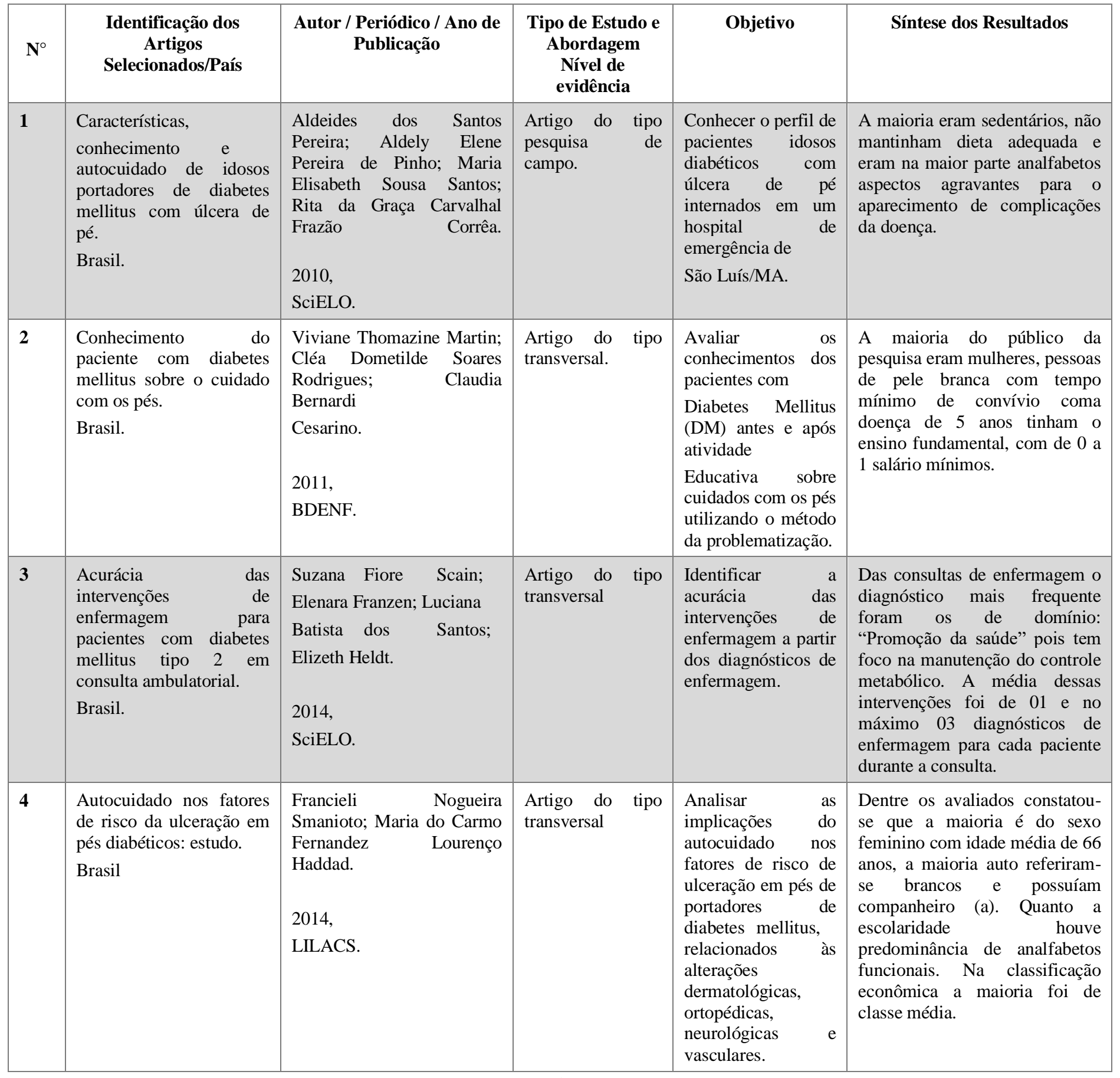




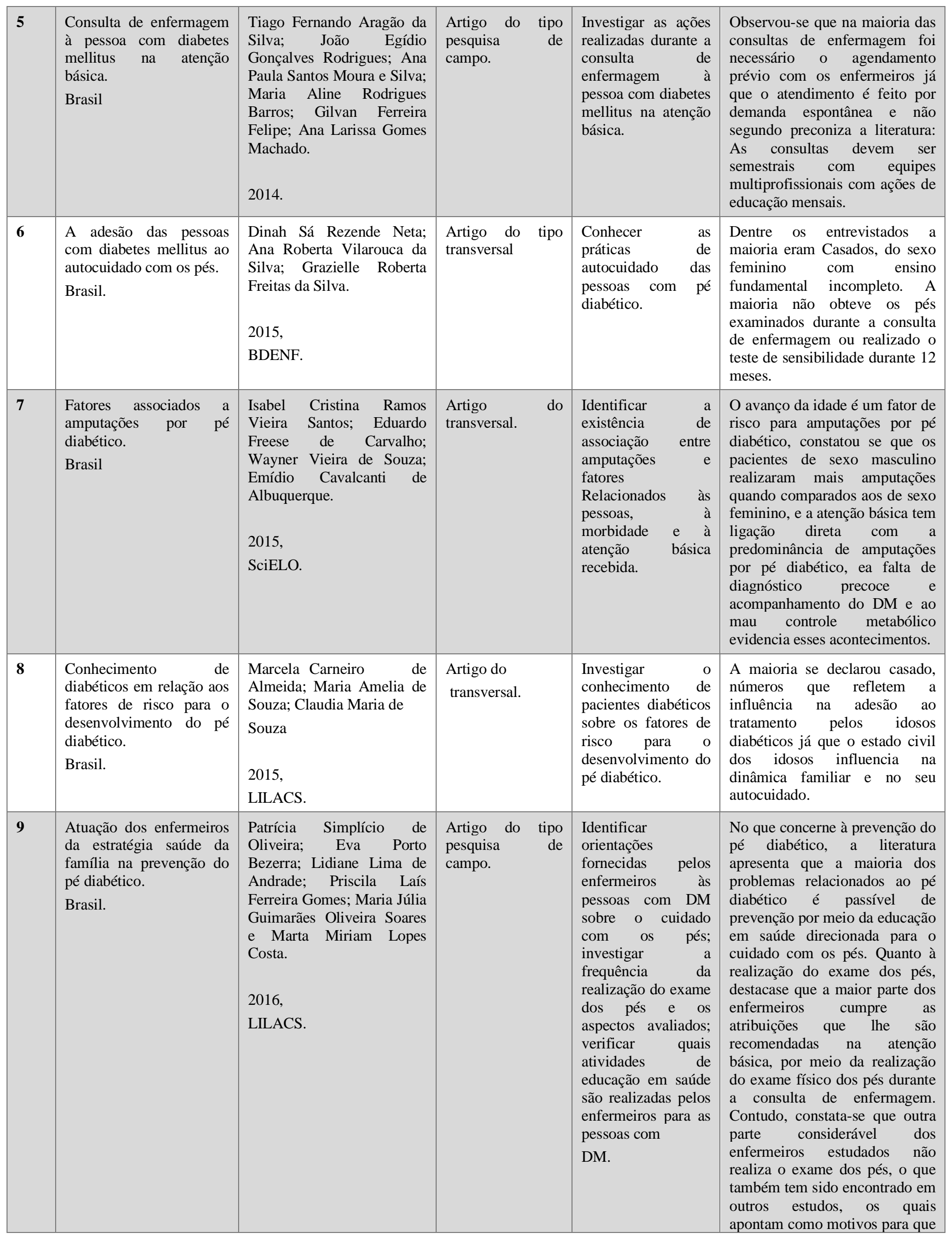




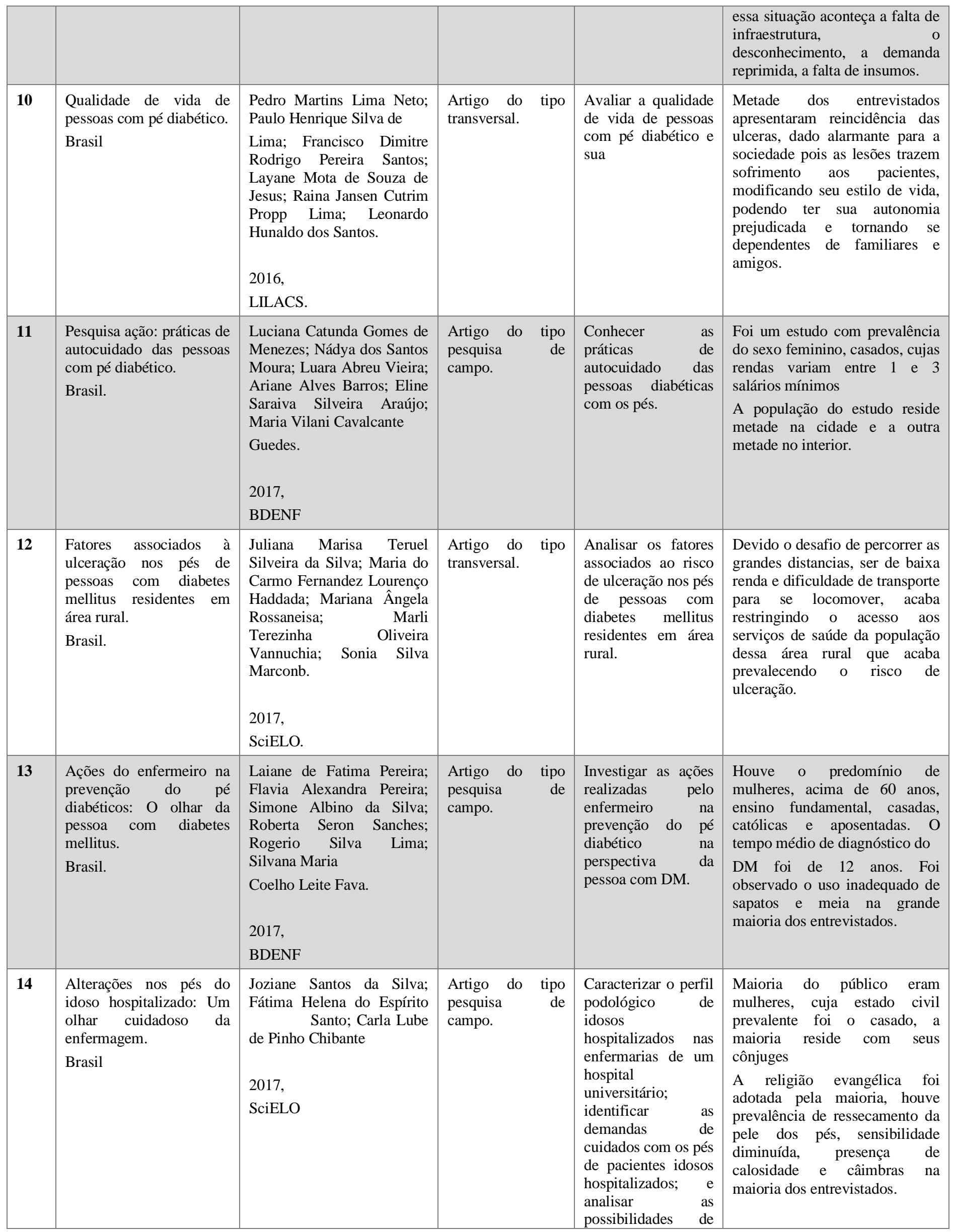




\begin{tabular}{|l|l|l|l|l|l|}
\hline & & & $\begin{array}{l}\text { atuação do } \\
\text { enfermeiro junto a } \\
\text { esses idosos. }\end{array}$ & \\
\hline
\end{tabular}

\subsection{Categorias Analisadas}

Fonte: Autores da pesquisa (2018).

\section{Categoria 1 - Caracterização do idoso com diabetes mellitus e a importância do autocuidado}

Nesta primeira categoria identificou-se que 06 artigos (40\%) abordam sobre o perfil e conhecimento do idoso a respeito do DM e as práticas de autocuidado realizada por estes, para não evolução do pé diabético. Dentre os 14 artigos selecionados para esta pesquisa, percebe-se que a maioria predominantemente dos idosos é mulher, com faixa etária entre 60 e 71 anos são casadas e possuem renda de até 3 salários mínimos.

A faixa etária evidencia que com o passar da idade esse público se torna mais suscetível a patologias crônicas com o aumento do processo de envelhecimento. A maioria ser mulher nos evidencia o fato denominado: feminização da velhice. As mulheres constituem a maioria da população idosa em todas as regiões do mundo, e as estimativas são de que as mulheres vivam, em média, de cinco a sete anos a mais que os homens (Almeida et.al., 2015).

Quanto ao estado conjugal, foi constatado nos artigos que a maioria dos idosos eram casados, aposentados e possuíam renda de até 3 salários-mínimos. O estado conjugal "casado" mostra que os mesmos consideram o vínculo conjugal como fator determinante para maiores cuidados com a doença. Já em relação ao nível de escolaridade, os idosos apresentam no máximo o ensino fundamental completo. Tal fato dificulta a compreensão das orientações que são feitas pela equipe de saúde, pois quanto menor a escolaridade, menor o acesso à informação e capacidade de compreensão e mostra-se como agravante para evolução do DM, haja vista que a falta ou pouco conhecimento sobre a fisiopatologia da doença faz com que essa se agrave e possa evoluir para o pé diabético (Silva, Santo \& Chibante, 2017).

Para Martins et al. (2011), é comprovado que o aumento crescente da prevalência de DM está diretamente ligado com o aumento da idade e que fatores como a obesidade e baixa escolaridade contribuem diretamente para elevar a prevalência de DM durante o envelhecimento. Observa-se ainda que a HAS e DM seguem como principais Doenças Crônicas Não Transmissíveis (DCNT) encontradas nos idosos e que geralmente descompensam e favorecem o surgimento de outras patologias, aumentando a demanda de internações hospitalares (Braga et.al., 2014).

Em 1991, Dorothea Orem definiu o autocuidado na sua Teoria do Déficit do Autocuidado de Enfermagem. Assim, para a teórica, autocuidado "é o desempenho ou a prática de atividades que os indivíduos realizam em seu benefício para manter a vida, a saúde e o bem-estar.” Este autocuidado é universal por abranger todos os aspectos vivenciais, não se restringindo às atividades de vida diária e às instrumentais (Santos et al., 2017).

Ainda para Santos et al. (2017), desenvolveu o seu projeto em três teorias inter-relacionadas, que são: a Teoria do Autocuidado; a Teoria do Déficit de Autocuidado e a Teoria dos Sistemas de Enfermagem. Orem reconhece o ser humano e o ambiente como uma única unidade e acredita que estes componentes influenciam-se reciprocamente. Crenças, antecedentes sociais e culturais, características pessoais e relação entre os profissionais de saúde e os clientes são alguns dos fatores que influenciam os comportamentos de autocuidado.

Para Neta et al. (2015), a participação ativa do paciente, por meio das atividades de autocuidado, torna-se a peça principal para o controle do diabetes mellitus (DM), uma vez que os pacientes e familiares são responsáveis por mais de 95\% do tratamento. As práticas de autocuidado devem envolver não apenas o idoso portador de diabetes, esse cuidado deve ser ampliador para todos os familiares onde o idoso está inserido.

A hidratação dos pés, procedimento que previne calosidades ou rachaduras, foi citado em apenas dois artigos analisados, dado que evidencia o auto índice de aparecimento destas patologias nos entrevistados visto que não há conhecimento sobre tal medida de cuidado. 
Diante este cenário, é de suma importância que o enfermeiro execute ações de educação em saúde para os pacientes sobre a importância do cuidado com os pés, nos quais devem se iniciar com a prática diária do autoexame dos pés. Portanto, melhorar o conhecimento da pessoa com DM, sobre os cuidados com os pés, é fundamental para minimizar ações adversas decorrentes do autocuidado ineficaz (Menezes et.al., 2017).

\section{Categoria 2 - Fatores de risco para o desencadeamento do pé diabético}

O envelhecimento é um fator de risco para o desenvolvimento do DM e suas complicações. Com o decorrer do envelhecimento, vários fatores implicam em uma série de alterações como: alterações no sistema vascular, neurológico e osteomuscular que predispõe ao aparecimento da doença vascular periférica e da neuropatia que são fatores que facilitam a predisposição para o aparecimento do pé diabético. (Smanioto et.al., 2014).

A prevalência da baixa escolaridade demostrou que a compreensão cognitiva é um fator que implica na predisposição do aparecimento do pé diabético visto que limita os indivíduos na obtenção de maiores entendimentos quanto ao cuidado e controle adequado da doença, a falta desse entendimento ou o desconhecimento sobre a patologia é considerada um obstáculo no cuidado ao idoso, pois a busca pelo tratamento só acontece quando esse paciente entende sobre sua patologia e a necessidade de controle (Martin, Rodrigues \& Cesarino, 2011).

Para Baggio et al. (2013), o baixo nível de escolaridade entre os idosos é um dos fatores que dificultam o entendimento, o conhecimento da causa e o cuidado precoce das úlceras que são essenciais para um bom prognóstico. Dessa forma, é de extrema importância que as informações e orientações que são repassadas pelo enfermeiro quanto á prevenção do pé diabético sejam captadas com clareza.

Segundo Almeida et al. (2013), idosos que recebem entre 01 e 02 salários-mínimos tem dificuldades em manter o tratamento, pois, a maioria desses idosos são chefes de família e acabam comprometendo a maioria de sua renda com contas e mantimentos para seus lares o que dificulta em sua maioria a adesão e a manutenção do tratamento visto que os impossibilitam de acesso a calçados específicos, alimentação adequada e até mesmo hábitos de higiene corretos.

Em outro estudo Silva et al. (2017), os entrevistados também relataram que o corte das unhas não é feito por profissionais capacitados, que acabam favorecendo as práticas de corte da unha inadequado, fica comprovado que o corte de forma arredondada favorece para o aparecimento de lesões nos cantos dos dedos e o uso de sapatos inadequados influenciam diretamente para o aparecimento de lesões e cortes.

Para Senteio et al. (2018), o uso de sapatos abertos, o corte inadequado das unhas, presença de rachaduras ou calos, movimento das articulações diminuídas, uso de sapatos apertados, são fatores que facilitam o aparecimento do pé diabético, e os mesmos fazem parte do processo do autocuidado.

Outro fator que foi analisado com frequência nos artigos estudados foi a perda da sensibilidade total ou parcial dos membros inferiores, essa perda associada a prática de escalda pés pode causar um enorme dano aos pés do paciente já que a sensibilidade alterada limita a percepção do paciente quanto a ferimentos e temperaturas elevadas, favorecendo o aparecimento de queimaduras que podem evoluir a lesões mais graves.

Para Silva et al. (2017), a vulnerabilidade da pele aliada a perda da sensibilidade tátil favorece o aparecimento de lesões, pois ausência da dor impedem que os idosos percebam lesões superficiais repetitivos que causam danos nos pés.

\section{Categoria 3 - Principais intervenções de enfermagem ao idoso portador de pé diabético}

A Consulta de Enfermagem (CE) é uma oportunidade ampla de desenvolvimento de práticas cuidativas, tais como: fortalecimento do vínculo, educação em saúde, avaliação multidimensional, identificação precoce de idosos frágeis ou em processo de fragilização, entre outras. Portanto, o enfermeiro tem papel fundamental nas respostas às necessidades de saúde da 
população na Atenção Básica e a CE aparece como uma estratégia de cuidado, além de um espaço de promoção da saúde e prevenção de agravos deste segmento da população (Silva \& Santos, 2014).

Mediante o exposto a NIC (Nursing Interventions Classification - Classificação das intervenções de Enfermagem) define intervenção de enfermagem como qualquer tratamento, que tenha por base o julgamento clínico e o conhecimento, que a enfermeira execute para melhorar os resultados do paciente (NIC, 2015).

Segundo Silva et al. (2014), as intervenções de enfermagem no sujeito com DM devem ser amplas e centradas no cenário da educação em saúde de modo que leve a prática eficaz do autocuidado, é necessária a busca de estratégias para a resolução de problemas específicos apresentados pela população, além de estimular incessantemente a continuidade do tratamento.

As intervenções de enfermagem foram descritas em 05 artigos (36,5\%), contudo, uma parte considerável dos enfermeiros estudados não realiza o exame dos pés e/ou não ensinam de forma eficaz, e a mesma tem que ser feita independentemente da presença de ulcerações, pois é a melhor forma de medida de cuidado para a prevenção de agravos. Ressalta-se que a consulta de enfermagem precisa ser sistemática, clara e objetiva, pois além de examinar o enfermeiro precisa repassar as orientações quanto a doença de forma que o idoso compreenda a necessidade de seguir o tratamento e manter a prática do autocuidado, prevenindo complicações mais severas como a amputação (Porto et al., 2016).

Segundo Oliveira et al. (2016), a orientação é uma ferramenta primordial, que permite ao enfermeiro estimular o autocuidado por meio de educação em saúde, pois proporciona ao paciente a consciência da importância de um tratamento eficaz além de estimular a disseminação das informações obtidas pelos pacientes e, maior participação nas decisões e atitudes voltadas à sua saúde.

Para Silva et al. (2016), o enfermeiro e o profissional responsável pelo cuidado técnico- científico e deve consolidar o elo com esses pacientes executando o exame físico criterioso que possa esclarecer possíveis riscos para o desenvolvimento de problemas nos pés, e ainda estar qualificado para assistir a esses problemas em sua integralidade.

Sendo assim, ações de educação em saúde que não valorizam o saber e as necessidades da pessoa com DM tornam-se ineficazes por não serem capaz de sensibilizar a pessoa para adoção de novos hábitos e estilo de vida. A educação em saúde como uma forma de cuidado deve envolver a participação da pessoa neste processo, dando a esta autonomia e possibilidade de problematizar sobre o processo saúde-doença-cuidado (Pereira et.al, 2017).

\section{Conclusão}

A partir da revisão de literatura, foi possível constatar que o cuidado com o pé diabético e a abordagem ao paciente diabético são complexos, pois requer uma intima colaboração e responsabilidade tanto dos pacientes, como dos profissionais, para evitar o desenvolvimento de possíveis complicações.

Por meio dos levantamentos foram observados que a maioria das intervenções dos enfermeiros é baseada em queixas dos pacientes e não em uma consulta sistematizada onde o mesmo poderia identificar possíveis riscos à saúde do paciente. Diante disto, reforça-se a necessidade de um atendimento onde além de ouvir também possa investigar e repassar o conhecimento, visto que a maioria dos idosos necessita de um acompanhamento em sua integralidade para que assim consigam prosseguir com o tratamento. Neste caso o enfermeiro precisa focar seu atendimento nessa população e suas necessidades para que o atendimento possa ser individualizado e harmonizado.

Foi observado ainda que as intervenções são realizadas na maioria das consultas de enfermagem, sendo de forma deficitária, assim, enfatiza-se que o enfermeiro, além de repassar informações de maneira clara e objetiva, precisa ensinar de modo que o idoso além de ouvir também entenda as informações para que assim se garanta um bom tratamento. Observou-se 
também que algumas dessas intervenções só foram feitas quando existia o risco ou doença instalada com possíveis agravos, visto que as mesmas também precisam ser feitas de modo que previnam danos.

Que a realização dessa revisão seja uma alerta para a sociedade acadêmica para que as intervenções de enfermagem não se limitem apenas em prescrições de doenças já instaladas e sim em ações e promoções de estreitamento entre profissional e paciente, já que o enfermeiro é o principal responsável por consolidar o elo entre equipe e paciente, além de estimular e ensinar esses idosos a serem detentores da responsabilidade pelo controle de sua própria doença, através de um processo colaboração e não apenas, fundamentalmente, prescritivo.

\section{Referências}

Almeida, A. V., Mafra, S. C. T., da Silva, E. P., \& Kanso, S. (2015). A Feminização da Velhice: em foco as características socioeconômicas, pessoais e familiares das idosas e o risco social. Textos \& Contextos (Porto Alegre), 14(1), 115-131. https://revistaseletronicas.pucrs.br/index.php/fass/article/download/19830/13313/

Braga, D. C., Bortolini, S. M., Mattia, M. B., \& Gehlen, B. (2014). Perfil dos pacientes encaminhados de uma estratégia saúde da família para um hospital geral, no município de Água Doce, Santa Catarina. Unoesc \& Ciência, 5(1), 109-14. https://core.ac.uk/download/pdf/235124204.pdf.

Baggio, S. C., de Lima Santos, A., Sales, C. A., \& Marcon, S. S. (2013). Percepção de pessoas com diabetes sobre a doença e os motivos de rehospitalização: estudo descritivo. Online Brazilian Journal of Nursing, 12(3), 501-10. http://objnursing.uff.br/index.php/nursing/article/view/4080/8367

Bardin, L. (2008). Análise de conteúdo. Lisboa: Edições 70; 1977. Correspondência: Daiane Dal Pai Rua Santana.

Barros, M. D. F. A., Mendes, J. C., Nascimento, J. A. D., \& Carvalho, A. G. C. D. (2012). Impacto de intervenção fisioterapêu tica na prevenção do pé diabético. Fisioterapia em Movimento, 25(4), 747-757. https://www.scielo.br/scielo.php?pid=S0103-51502012000400007\&script=sci_arttext\&tlng=pt.

Cubas, M. et al. (2013). Pé diabético: orientações e conhecimento sobre cuidados preventivos. Fisioter. Mov., Curitiba, 26(3), 647-655, jul./set. http://www.scielo.br/pdf/fm/v26n3/a19v26n3.pdf.

Caiafa, J. S., Castro, A. A., Fidelis, C., Santos, V. P., Silva, E. S. D., \& Sitrângulo Jr, C. J. (2011). Atenção integral ao portador de pé diabético. Jornal vascular brasileiro, 10(4), 1-32. https://www.scielo.br/scielo.php?pid=S1677-54492011000600001\&script=sci_arttext\&tlng=pt

da Silva, C. A. M., de Sousa Pereira, D., da Costa Almeida, D. S., \& Venâncio, M. I. L. (2014). Pé diabético e avaliação do risco de ulceração. Revista de Enfermagem Referência, 4(1), 153-161. https://www.redalyc.org/pdf/3882/388239971010.pdf

da Silva, J. M. T. S., Haddad, M. D. C. F. L., Rossaneis, M. A., Vannuchi, M. T. O., \& Marcon, S. S. (2017). Fatores associados à ulceração nos pés de pessoas com diabetes mellitus residentes em área rural. Revista Gaúcha de Enfermagem, 38(3). http://www.scielo.br/scielo.php?pid=S198314472017000300411\&script=sci_abstract \&tlng=pt>

De Oliveira, P. S., Bezerra, E. P., De Andrade, L. L., Gomes, P. L. F., Soares, M. J. G. O., \& Costa, M. M. L. (2016). Atuação do enfermeiros da Estratégia Saúde da Família na prevenção do pé diabético Revista de Pesquisa: Cuidado é Fundamental Online, 8(3), 4841-4849.http://www.seer.uniri o.br/index.php/cuidadofundamental/article/view/4398/0>.

de Sousa, L. S. N., Rodrigues, M. T. P., Mascarenhas, M. D. M., \& da Silva, A. R. V. (2017). Conhecimento do enfermeiro sobre a prevenção do pé diabético: revisão integrativa da literatura. Revista Brasileira em Promoção da Saúde, 30(3). https://periodicos.unifor.br/RBPS/article/download/6602/pdf

dos Santos Pereira, A., de Pinho, A. E. P., Santos, M. E. S., \& Corrêa, R. D. G. C. F. (2011). Características, conhecimento e autocuidado de idosos portadores de diabetes melittus com úlcera de pé Revista de Pesquisa em Saúde, 11(2). http://www.periodicoseletronicos.ufma.br/index .php/revistahuufma/article/view/547>.

de Menezes, L. C. G., dos Santos Moura, N., Vieira, L. A., Barros, A. A., Araújo, E. S. S., \& Guedes, M. V. C. Pesquisa ação: práticas de autocuidado das pessoas com pé diabético. http://bases.bireme.br/cgibin/wxislind.exe/iah/online/?IsisScript=iah/iah.xis\&src=google\&base=BDENF\&lang=p>.

De Oliveira, P. S., Bezerra, E. P., De Andrade, L. L., Gomes, P. L. F., Soares, M. J. G. O., \& Costa, M. M. L. (2016). Atuação do enfermeiros da Estratégia Saúde da Família na prevenção do pé diabético Revista de Pesquisa: Cuidado é Fundamental Online, 8(3), 4841-4849. http://www.seer.unirio.br/i ndex.php/cuidadofundamental/article/view/4398/0>

Diretrizes, S. B. D. (2015). Epidemiologia e prevenção do diabetes mellitus.

de Almeida, M. C., de Souza, M. A., \& de Souza, C. M. (2013). Conhecimento de diabéticos em relação aos fatores de risco para o desenvolvimento do pé diabético. Revista de Ciências da Saúde Nova Esperança, 11(3), 4-12. http://revistanovaesperanca.com.br/index.php/revistane/article/download/428/322.

dos Reis Carvalho, E. (2016). A importância da assistência de enfermagem ao paciente portador de diabetes mellitus: revisão bibliográfica. Revista Iniciare, 1(1). http://revista2.grupointegrado.br/revista/index.php/iniciare/article/download/2318/852.

Ferreira, D. L., Fonseca, E. C. R., Lucas, A. L. R., Silva, A. C. F., Lenci, S. S., Silva, S. G. F., \& de Resende, E. A. M. R. (2020). O efeito da orientação preventiva multiprofissional em pacientes com diabetes mellitus. Revista Eletrônica Acervo Saúde, (45), e2381-e2381. https://acervomais.com.br/index.php/saude/article/download/2381/1733.

Lima, P. M. N. et al. (2016). Qualidade De Vida Da Pessoa Com Pé Diabético. Revista Rene. http://www.periodicos.ufc.br/rene/article/view/2996> 
Marques, M. B., Coutinho, J. F. V., Martins, M. C., Lopes, M. V. D. O., Maia, J. C., \& Silva, M. J. D. (2019). Intervenção educativa para a promoção do autocuidado de idosos com diabetes mellitus. Revista da Escola de Enfermagem da USP, 53. https://www.scielo.br/scielo.php?script=sci_arttext\&pid=S0080$62342019000100490 \&$ tlng=pt.

Martin, V. T., Rodrigues, C. D. S., \& Cesarino, C. B. (2011). Conhecimento do paciente com diabetes mellitus sobre o cuidado com os pés. Rev. enferm. UERJ, 621-625. http://www.facenf.uerj.br/v19n4/v19n4a20.pdf>.

Ministério da saúde (2013). Departamento de Atenção Básica. Estratégia para o Cuidado de Pessoa com Doenças Crônicas Diabetes Mellitus. BrasíliaDF,160p. http://bvsms.saude.gov.br/bvs/publicacoes/estrategias_cuidado_pessoa_diabetes_mellitus_cab36.pdf

Rezende Neta, D. S., Silva, A. R. V. D., \& Silva, G. R. F. D. (2015). Adesão das pessoas com diabetes mellitus ao autocuidado com os pés. Revista Brasileira de Enfermagem, 68(1), 111-116. http://www.scielo.br/scielo.php?pid=S003471672015000100111\&script=sci_abstract\&tlng=pt $>$.

Pereira, L. D. F., Paiva, F. A. P., Silva, S. A. D., Sanches, R. S., Lima, R. S., \& Fava, S. M. C. L. (2017). Ações do enfermeiro na prevenção do pé diabético: o olhar da pessoa com diabetes mellitus. Rev. pesqui. cuid. fundam.(Online), 1008-1014. http://bases.bireme.br/cgibin/wxislind.exe/iah/ online/?IsisScript=iah/iah.xis\&src=google\&base=BDENF\&lang=p\&nextAction=lnk\&exprSearch=31878\&indexSearch=ID $>$.

Rezende Neta, D. S., Silva, A. R. V. D., \& Silva, G. R. F. D. (2015). Adesão das pessoas com diabetes mellitus ao autocuidado com os pés. Revista Brasileira de Enfermagem, 68(1), 111-116. http://www.scielo.br/scielo.php?pid=S003471672015000100111\&script=sci_abstract\&tlng=pt $>$.

Pereira, L. D. F., Paiva, F. A. P., Silva, S. A. D., Sanches, R. S., Lima, R. S., \& Fava, S. M. C. L. (2017). Ações do enfermeiro na prevenção do pé diabético: o olhar da pessoa com diabetes mellitus. Rev. pesqui. cuid. fundam.(Online), 1008-1014. http://bases.bireme.br/cgibin/wxislind.exe/iah/o nline/?IsisScript=iah/iah.xis\&src=google\&base=BDENF\&lang=p\&nextAction=lnk\&exprSearch=31878\&indexSearch=ID > .

Silva, A. B. D., Engroff, P., Sgnaolin, V., Ely, L. S., \& Gomes, I. (2016). Prevalência de diabetes mellitus e adesão medicamentosa em idosos da Estratégia Saúde da Família de Porto Alegre/RS. Cadernos Saúde Coletiva, 24(3), 308-316. https://www.scielo.br/scielo.php?pid=S1414462X2016000300308\&script=sci_arttext

Silva, K. M., \& dos Santos, S. M. A. (2014). A consulta de enfermagem ao idoso na estratégia saúde da família: desafios e possibilidades. Ciência, Cuidado e Saúde, 13(1), 49-57. http://ojs.uem.br/ojs/index.php/CiencCuidSaude/article/viewFile/20128/pdf_112>

Silva, T. F. et al. (2014). Consulta de Enfermagem à Pessoa com Diabetes Mellitus na Atenção Básica. Reme, jul/set; 18(3): 710-716. www.reme.org.br/artigo/detalhes/957>.

Santos, I. C. R. V., de Carvalho, E. F., de Souza, W. V., \& de Albuquerque, E. C. (2015). Fatores associados a amputações por pé diabético. Jornal Vascular Brasileiro, 14(1), 37-45. https://www.redalyc.org/pdf/2450/245038353007.pdf.

Senteio, J. D. S., Teston, E. F., Costa, M. A. R., Soares, V. D. S., \& Novakowski, D. (2018). Prevalência de fatores de risco para o desenvolvimento de pé diabético. Rev. pesqui. cuid. fundam.(Online), 919-925. https://pesquisa.bvsalud.org/portal/resource/pt/biblio-915523.

Scain, S. F., Franzen, E., Santos, L. B. D., \& Heldt, E. (2013). Acurácia das intervenções de enfermagem para pacientes com diabetes mellitus tipo 2 em consulta ambulatorial. Revista Gaúcha de Enfermagem, 34(2), 14-20. www.scielo.br/scielo.php?pid=S198314472013000200002\&script=sci...tlng >.

Smanioto, F. N., Haddad, M. D. C. F. L., \& Rossaneis, M. A. (2014). Autocuidado nos fatores de risco da ulceração em pés diabéticos: estudo transversal. Online Brazilian Journal of Nursing, 13(3), 343-352. www.objnursing.uff.br/index.php/nursing/article/viewFile/4680/pdf_178>.

Ursi, E. S., \& Gavão, C. M. (2006). Prevenção de lesões de pele no perioperatório: revisão integrativa da literatura. Revista Latino-Americana de Enfermagem, 14(1), 124-131. https://www.scielo.br/scielo.php?pid=S0104-11692006000100017\&script=sci_arttext. 